\title{
Desenvolvimento Local e Arranjos Produtivos Locais: uma revisão sistemática da literatura
}

\author{
Local Development and Clusters: a systematic literature review
}

\section{Développement Locale et Arrangements Productifs Locaux: une revue systématique de la littérature}

\author{
Desarrollo Local y Arreglos Productivos Locales: uma revisión sistemática de la literatura
}

\author{
Márcia Freire de Oliveira* \\ (marciafreire@fagen.ufu.br) \\ Dante Pinheiro Martinelli** \\ (dantepm@usp.br)
}

Recebido em 10/02/2013; revisado e aprovado em 23/02/2013; aceito em 29/03/2013

\begin{abstract}
Resumo: Este artigo tem como objetivo avaliar, através de revisão sistemática, a produção bibliográfica a respeito do desenvolvimento local em arranjos produtivos locais. A partir de vinte e quatro artigos selecionados, os resultados mostram que é possível agrupá-los nos seguintes temas, relacionados ao desenvolvimento local: A) Uso de indicadores, métodos e técnicas para avaliação de arranjos produtivos; B) Impacto de arranjos produtivos de setores específicos; C) Desenvolvimento e promoção de arranjos produtivos.
\end{abstract}

Palavras-chave: Desenvolvimento Local. Arranjos Produtivos Locais. Revisão Sistemática.

Abstract: This article aims to evaluate, through a systematic review, the bibliographic production regarding local development in clusters. From the twenty four articles selected the results show that it is possible to group them in the following themes, related to the local development: A) Use of indicators, methods and techniques for evaluation of clusters; B) Impact of clusters of specific sectors; C) Development and promotion of clusters.

Key words: Local Development. Clusters. Systematic review.

Résumé: Cet article a comme objectif d'évaluer, à travers la revue systématique, la production bibliographique sur le développement local en arrangements productifs locaux. À partir de vingt-quatre articles selectionés les résultats montrent qu'il est possible de les grouper selon les thémes liés au développement local, comme suit: A) L'utilisation d'indicateurs, de méthodes et de techniques pour évaluer les arrangements productifs; B) L'impact des arrangements productifs des secteurs spécifiques; C) Le développement et la promotion des arrangements productifs.

Mots-clés: Développement Local. Arrangements Productifs. Revue Systématique.

Resumen: Este artículo tiene como objetivo evaluar a través de una revisión sistemática la producción bibliográfica sobre el desarrollo local en las agrupaciones locales. De veinte y cuatro artículos seleccionados resultados muestran que es posible agruparlos en los siguientes temas, A) El uso de indicadores, métodos y técnicas para la evaluación de los arreglos de producción B) Impacto de arreglos de producción en sectores específicos; C) Desarrollo y promoción de los arreglos productivos.

Palabras clave: Desarrollo Local. Arreglos Productivos Locales. Revisión Sistemática.

\section{Introdução}

Os estudos sobre aglomerações produtivas locais vêm ganhando importância na literatura, sendo um tema bastante debatido em estudos nacionais e internacionais. As empresas, em sua maioria micro e pequenas, quando aglomeradas, isto é, próximas espacialmente, podem ganhar vantagens que não alcançariam se agissem isoladamente. Além disso, os benefícios gerados a partir da proximidade territorial das empresas vão além do contexto de cada organização, podendo contribuir para o desenvolvimento local das regiões onde esses arranjos estão localizados.

Os arranjos produtivos locais (APLs) são aglomerações territoriais de agentes econômicos, políticos e sociais - com foco em um conjunto específico de atividades econômicas - que apresentam vínculos mesmo que incipientes. Em geral, envolvem a participação e a interação de empresas - que podem ser desde produtoras de bens e serviços finais até fornecedoras de insumos e equipamentos,

\footnotetext{
* Universidade Federal de Uberlândia (UFU), Uberlândia, MG, Brasil.

** Faculdade de Economia, Administração e Contabilidade - Universidade de São Paulo (FEARP-USP), Ribeirão Preto, SP, Brasil.
} 
consultorias, clientes e outros - e suas diversas formas de representação e associação. Incluem também outras instituições públicas e privadas voltadas para: capacitação de recursos humanos; pesquisa, desenvolvimento e engenharia; política, promoção e financiamento (CASSIOLATO; LASTRES, 2003).

Segundo Mattos (2008), o desenvolvimento local abrange, pelo menos, três dimensões: uma econômica, caracterizada por um sistema específico de produção capaz de assegurar, aos empresários locais, o uso eficiente dos fatores produtivos e a melhoria dos níveis de produtividade que lhes garantem competitividade; uma outra, sociocultural, na qual os atores econômicos e sociais se integram às instituições locais e formam um denso sistema de relações, que incorpora os valores da sociedade ao processo de desenvolvimento; e uma última, que é política e se materializa em iniciativas locais, possibilitando a criação de um entorno local que incentiva a produção e favorece o desenvolvimento.

O termo APL, não é novo, porém sua apropriação como política de Estado para o desenvolvimento local e regional no Brasil, seja em indústrias tradicionais ou tecnológicas, o é (ULTRAMARI; DUARTE, 2011). Isbasoiu (2007) coloca que os APLs exercem um papel significativo no desenvolvimento local, gerando benefícios como: melhoria financeira e tecnológica das empresas, geração de oportunidades de trabalho, criação de riqueza e renda e maior nível de crescimento econômico global, quando comparado às regiões onde as empresas atuam de forma isolada. Assim, os APLs são reconhecidos por terem um papel significativo no desenvolvimento econômico regional e na melhoria da qualidade de vida nas localidades onde estão instalados.

Dessa forma, coloca-se a seguinte pergunta de pesquisa: qual a produção bibliográfica que trata do desenvolvimento local gerado por meio de arranjos produtivos locais?

Buscando responder tal questão, este artigo tem como objetivo avaliar, através de revisão sistemática, a produção bibliográfica a respeito do desenvolvimento local em arranjos produtivos locais nas bases de dados ABI/Inform, Scopus e SciELO, produzida de 2007 a 2012.

A revisão sistemática é um método baseado na revisão da literatura que foca uma pergunta definida de forma clara, para a qual são identificados, selecionados e avaliados artigos buscando a síntese de evidências importantes, envolvendo a aplicação de critérios explícitos e procedimentos padronizados e rigorosos (FUCHS; PAIM, 2010).

Os estudos de revisão sistemática são bastante utilizados em pesquisas em áreas de saúde, porém pouco difundidos na área de gestão. Segundo Tranfield, Denyer e Smart (2003), os estudos de revisão sistemática podem auxiliar os estudos de gestão contribuindo principalmente com a melhoria do rigor metodológico das pesquisas.

O presente artigo, além desta introdução está dividido em sete seções. Na primeira, o método usado para a realização da pesquisa é detalhado; na segunda, os resultados são apresentados e desdobrados posteriormente na terceira, quarta e quinta seções, denominadas respectivamente: uso de indicadores, métodos e técnicas para avaliação de arranjos produtivos; impacto de arranjos produtivos de setores específicos; e desenvolvimento e promoção de arranjos produtivos. As considerações finais são descritas na sexta seção e, por fim na sétima seção são feitos os agradecimentos.

\section{Método}

A pesquisa dos artigos foi realizada mediante a busca eletrônica nas bases de dados Abi/Inform, Scopus e SciELO. Foram utilizados os seguintes critérios de inclusão para a realização da busca: I) idioma de publicação - artigos publicados na íntegra em língua inglesa, francesa ou portuguesa; II) ano de publicação - artigos publicados entre 2007 e 2012, totalizando um período de 6 anos.

Para as bases de dados Abi/Inform e Scopus utilizaram-se cruzamentos dos seguintes descritores: local development and cluster; regional development and cluster; territorial development and cluster; local development and agglomeration; regional development and agglomeration; territorial development and agglomeration. Para a base de dados SciELO, os cruzamentos dos seguintes descritores foram utilizados: desenvolvimento local e cluster; desenvolvimento regional e cluster; desenvolvimento territorial e cluster; desenvolvimento local e arranjo; desenvolvimento regional 
e arranjo; desenvolvimento territorial e arranjo; desenvolvimento local e aglomeração; desenvolvimento regional e aglomeração e; desenvolvimento territorial e aglomeração.

Nas bases de dados Abi/Inform e SciELO, a busca foi feita pelo título e resumo; na base Scopus, a busca foi feita no título e artigo completo, pois não havia opção de fazer a busca apenas pelo resumo. Foram encontrados 10 artigos na Abi/Inform, 29 artigos na Scopus e 11 artigos no SciELO, totalizando 50 artigos. Realizou-se uma leitura seletiva dos resumos, o que resultou no descarte de 13 artigos (por exemplo, artigos em que a palavra cluster estava relacionada ao método estatístico de análise, e não a cluster como aglomerado). Posteriormente realizouse a leitura completa dos 37 artigos restantes, descartando-se mais 13 artigos (por exemplo, artigos que apenas faziam menção a clusters ou arranjos, mas que abordavam apenas o contexto de redes de empresas).

Assim, o corpus que representou o material de análise foi constituído por 24 artigos. Foi feita uma segunda leitura completa dos artigos tabulando-os nas seguintes dimensões: nome do artigo; autores; periódico; ano de publicação; objetivos; base teórica de referência; método; e principais resultados. Dessa maneira, foi possível obter uma análise dos estudos selecionados, o que proporcionou um panorama geral da produção científica nacional e internacional sobre desenvolvimento local e regional no contexto dos arranjos produtivos locais. Após a tabulação, levantaramse os temas que emergiram da leitura e análise dos artigos, agrupando-os de acordo com a semelhança entre os estudos.

\section{Resultados}

Os artigos constituintes do corpus de análise foram publicados em vinte periódicos, observando-se uma grande diversidade de revistas que publicaram sobre o tema desenvolvimento local no contexto dos arranjos produtivos locais. A maioria dos periódicos, dezessete, publicou apenas um artigo relacionado ao tema, com exceção da Revista Brasileira de Gestão e Desenvolvimento Regional, com três artigos publicados, e as revistas Economic Development Quartely e Urban Studies, com dois artigos publicados em cada uma.

Em relação ao ano de publicação dos artigos, quatro artigos foram publicados em 2007; quatro, em 2008; dois, em 2009; quatro, em 2010 e cinco, tanto em 2011 quanto em 2012. Portanto percebe-se que houve certa homogeneidade por ano na quantidade de artigos publicados sobre o tema pesquisado neste artigo, exceto no ano de 2009.

Em se tratando do número de autores, seis artigos foram publicados por um autor cada; sete artigos por dois autores cada; oito artigos por três autores cada; dois artigos por quatro autores cada e; um artigo foi publicado por nove autores.

Em se tratando do número de autores por publicação, seis artigos foram publicados por um único autor; sete artigos, por dois; oito artigos, por três; dois artigos, por quatro; e um artigo foi publicado por nove autores.

Os artigos pesquisados foram agrupados segundo os seguintes temas, relacionados ao desenvolvimento local: A) Uso de indicadores, métodos e técnicas para avaliação de arranjos produtivos; B) Impacto de arranjos produtivos de setores específicos; C) Desenvolvimento e promoção de arranjos produtivos. O Quadro 1, mostra a relação dos autores com os temas. O tema B foi o que apresentou o menor número de artigos, apenas três. Já o tema $C$ foi o que apresentou o maior número de artigos, onze, e o tema A apresentou dez artigos. 


\begin{tabular}{|c|c|c|c|}
\hline Tema & $\begin{array}{l}\text { Uso de indicadores, métodos } \\
\text { e técnicas para avaliação de } \\
\text { arranjos produtivos }\end{array}$ & $\begin{array}{l}\text { Impacto de arranjos } \\
\text { produtivos de setores } \\
\text { específicos; }\end{array}$ & $\begin{array}{l}\text { Desenvolvimento e pro- } \\
\text { moção de arranjos produ- } \\
\text { tivos }\end{array}$ \\
\hline Autores & $\begin{array}{l}\text { Scholz e Stauffacher (2007); } \\
\text { Fromhold-Eisebith e Eisebith } \\
\text { (2008); Pinheiro, Sobreira e } \\
\text { Rapini (2008); Galdámez, } \\
\text { Carpinetti e Gerolamo (2009); } \\
\text { Brito et al. (2010); Olivares } \\
\text { e Dalcol (2010); Li, Li e Liu } \\
\text { (2011); Tigre et. al. (2011); } \\
\text { Lübeck, Wittman e Silva (2012); } \\
\text { Junior Marini e Silva (2012). }\end{array}$ & $\begin{array}{l}\text { Giarratani, Gruver e } \\
\text { Jackson (2007); Sable } \\
\text { (2007); Oliveira e Ali } \\
\text { (2011). }\end{array}$ & $\begin{array}{l}\text { Wei, Li e Wang (2007); Mason, } \\
\text { Castleman e Parker (2008); } \\
\text { Treado e Giarratani (2008); } \\
\text { Czepczynski (2009); Franz e } \\
\text { Hornych (2010); Parker (2010); } \\
\text { Chou et al. (2011); Ranjith } \\
\text { e Widner (2011); Bellandi } \\
\text { e Lombardi (2012); Cunha, } \\
\text { Passador e Passador (2012); } \\
\text { Quandt (2012). }\end{array}$ \\
\hline
\end{tabular}

Quadro 1 - Relação de autores e temas

Fonte: Elaborado pelos autores.

Nos três subtítulos seguintes, serão detalhados os artigos relacionados a cada tema.

\section{Uso de indicadores, métodos e técnicas para avaliação de arranjos produtivos}

Os autores Scholz e Stauffacher (2007) procuraram compreender o caráter interativo do aprendizado regional, investigando os prérequisitos de tal processo e introduzindo um método, denominado ADN, para promover e iniciar clusters ou processos de aprendizado regional. O método foi aplicado em três clusters suíços dos setores têxtil, laticínios e serraria, envolvendo: (a) a construção de estratégias de negócios regionais e levantamento de variáveis dos clusters por meio de análise de cenário; (b) avaliação multicritério dessas variáveis e; (c) um sistema multiprocesso de consulta aos diversos agentes dos clusters sobre formas de cooperação horizontal e vertical. Como resultado da aplicação do método, ocorreu um processo de aprendizagem regional, ainda inicial, entre as indústrias, governo e instituições de pesquisa, com o objetivo final de se avançar para uma forma de desenvolvimento sustentável.

Fromhold-Eisebith e Eisebith (2008) discutiram questões gerais de avaliação de clusters e propuseram um modelo sistêmico (que engloba a análise do cluster e seu efeito para as empresas, para o cluster como um todo e para a economia regional) para avaliá-los. Eles realizaram uma pesquisa em dois clusters automotivos, um alemão e outro austríaco. Os resultados indicaram que ganhos advindos dos clusters pouco impactam o desempenho das empresas. Em termos coletivos, iniciativas criaram novos contatos e ativos intangíveis, mas de difícil sustentabilidade. A contribuição impacto da promoção de clusters parece ser menos significante e estruturalmente diferente do que é comumente considerado pela literatura.

Pinheiro, Sobreira e Rapini (2008) utilizaram a técnica de componentes principais e a matriz insumo-produto para identificar e investigar as aglomerações produtivas no setor de construção civil do estado do Pará, sustentados na ideia de que estímulos voltados a regiões onde existem aglomerações de empresas podem alavancar o desenvolvimento local pela atração de investimentos, tecnologia, mão de obra qualificada, instituições e agentes políticos, sociais e econômicos. Os autores verificaram a existência de quatro aglomerações no estado e um baixo potencial do setor de construção civil em gerar impactos sobre a economia paraense.

Já Galdámez, Carpinetti e Gerolamo (2009) propuseram um sistema de avaliação de desempenho para dar suporte ao processo de gestão de desempenho de APL. O sistema foi construído através de pesquisas de campo nos APLs de Ibitinga (indústria de cama, mesa e banho) e Jaú (calçados femininos). As perspectivas e indicadores de desempenho utilizadas nos APLs são apresentadas no Quadro 2. 


\begin{tabular}{|c|c|c|}
\hline Perspectiva de Desempenho & Indicadores APL de Ibitinga & Indicadores APL de Jaú \\
\hline Desempenho das empresas & $\begin{array}{l}\text { Preço médio de venda dos } \\
\text { produtos } \\
\text { Valor adicionado das pessoas } \\
\text { ocupadas } \\
\text { Custo total da empresa negócio } \\
\text { Lucratividade das PMEs }\end{array}$ & $\begin{array}{l}\text { Preço médio do calçado por } \\
\text { coleção } \\
\text { Produtividade do funcionário por } \\
\text { coleção } \\
\text { Pares de calçados produzidos e } \\
\text { comercializados por coleção }\end{array}$ \\
\hline $\begin{array}{l}\text { Resultados econômicos e } \\
\text { sociais }\end{array}$ & $\begin{array}{l}\text { Pessoas ocupadas } \\
\text { Pessoas qualificadas }\end{array}$ & $\begin{array}{l}\text { Pessoas ocupadas } \\
\text { Pessoas qualificadas }\end{array}$ \\
\hline Eficiência coletiva & Compra coletiva de fibra & Compra coletiva de cestas básicas \\
\hline Capital social & $\begin{array}{l}\text { Porcentagem de empresas } \\
\text { envolvidas nas ações de coop- } \\
\text { eração }\end{array}$ & $\begin{array}{l}\text { Empresas envolvidas nas ações } \\
\text { de cooperação }\end{array}$ \\
\hline Meio ambiente & & Coleta do resíduo industrial \\
\hline
\end{tabular}

Quadro 2 - Perspectivas e indicadores de desempenho dos APLs de Ibitinga e Jaú.

Fonte: Adaptado de Galdámez, Carpinetti e Gerolamo (2009).

A pesquisa mostrou que a medição de desempenho integrado a um processo de melhoria contínua promove a gestão colaborativa, aperfeiçoa o processo de tomada de decisão e apoia o desenvolvimento de ações de melhoria e mudança empresarial, pois identifica lacunas de desempenho. As informações obtidas com a medição de desempenho podem direcionar investimentos e implementação de políticas de desenvolvimento econômico, industrial e social por instituições governamentais, universidades, setores privados, agentes locais e as empresas do arranjo (GALDÁMEZ; CARPINETTI; GEROLAMO, 2009).

Brito et al. (2010) procuraram identificar a relação da aglomeração de firmas de uma mesma atividade econômica, do setor industrial paulista, na taxa de crescimento do emprego local, comparando municípios que possuem aglomerado com os que não possuem. A pesquisa utilizou modelagem multinível para medir o crescimento. Houve uma relação positiva entre as aglomerações e o crescimento de emprego, e as firmas localizadas na aglomeração apresentaram crescimento maior do que suas concorrentes localizadas fora de aglomerados.

Olivares e Dalcol (2010), por sua vez, apresentaram um sistema de indicadores para mensurar o grau de contribuição que os APLs assumem em relação ao desenvolvimento local e regional, visando auxiliar no processo de tomada de decisão por parte de formuladores de políticas e ações de promoção local. Os indicadores, divididos nos aspec- tos: socioeconômico; inovação; cooperação; geográfico; e promoção de ações e políticas foram aplicados em dois aglomerados, um de confecções e o outro de software, localizados em Petrópolis, RJ.

A pesquisa mostrou que o aglomerado de software possui um maior grau de contribuição para o desenvolvimento local e regional, embora não tão expressivo que o aglomerado de confecções, para o município de Petrópolis. Os autores sugerem que gestores e promotores de políticas públicas identifiquem os indicadores que tiveram baixa pontuação e adotem estratégias para melhoria dos índices (OLIVARES; DALCOL, 2010).

Li, Li e Liu (2011) analisaram, através da técnica de análise de redes sociais, as redes e sua evolução, procurando compreender o desenvolvimento industrial e regional de clusters de áreas rurais, menos desenvolvidas. Os autores pesquisaram o cluster da indústria de fitas localizado na área rural da aldeia de Narizhuang, no condado de Yucheng, China.

Comparado às tradicionais áreas costeiras chinesas ou aos clusters de alta tecnologia, a evolução de redes em clusters de indústrias tradicionais, em áreas rurais mais atrasadas e menos desenvolvidas, conta com recursos endógenos e segue princípios de autoorganização. Quatro tipos de redes foram identificados: spin-off; consultoria; comunicação; e cooperativa. Ao longo do tempo de desenvolvimento do cluster, este passou por quatro estágios: redes familiares (1978-1995), redes de divisão interna de produção (19952000); redes de inovação local (2001-2005) e 
redes de cadeia global (2005...), que exerceram diferentes papéis no desenvolvimento do cluster. Nesse processo, o número de nós da rede aumentou, os tipos de atores se diversificaram, as relações entre eles se estreitaram e as empresas do cluster foram se adaptando lentamente, de forma que as redes evoluíram naturalmente (ERLING; XIAOJIAN; ZHIGAO, 2011).

Tigre et al. (2011) buscaram levantar questões chaves relacionadas à atratividade para empresas de software de diferentes cidades na Argentina e no Brasil e seus impactos no desenvolvimento local; e propor uma taxonomia para agrupar clusters de acordo com o segmento dominante de negócios, origem do capital e escopo de operações.

Os resultados do estudo de Tigre et al. (2011) apontaram que cidades que são hábeis em atrair a indústria de software e serviços de informação apresentam uma combinação de empregados altamente capacitados, universidades de qualidade, ambiente institucional amigável, e governos locais que buscam desenvolver a indústria ativamente. Entretanto tal atração não garante o fluxo de conhecimento, uma vez que a transmissão de tecnologia para as firmas locais depende das relações entre diferentes agentes. Então, as perspectivas para o desenvolvimento local dependem da forma como os APLs são institucionalizados e geridos.

O artigo de Lübeck, Wittman e Silva (2012) teve como objetivo demonstrar a necessidade de se conjugar métodos e variáveis para traçar um quadro da situação produtiva territorial de aglomerados por meio da análise do capital social, redes de empresas, inovações, políticas públicas, gini locacional e indicadores econômicos. O estudo apontou que é possível diferenciar APLs de SLPIs pelo grau de maturidade da aglomeração. Nos APLs a intensidade de interação entre os agentes e a inovação é ainda insipiente, sendo que o aumento do capital social pode ser uma fonte importante de inovação, contribuindo para a geração de vantagens competitivas e de melhores possibilidades de desenvolvimento interno e aproveitamento de oportunidades oferecidas por agentes endógenos e exógenos locais e regionais.
Por fim, através do método bibliográfico, Junior Marini e Silva (2012) analisaram as inter-relações dos arranjos produtivos locais com o contexto do desenvolvimento regional, propondo uma matriz para compreender estas inter-relações através dos principais componentes que formam a estrutura dos APLs, como políticas públicas, ações conjuntas, governança local etc.; e as seguintes dimensões territoriais: social; econômica; ambiental; espacial; cultural; política e institucional.

Fazendo um análise geral dos artigos relacionados a este tópicos verifica-se que alguns deles propõem métodos/indicadores/técnicas de avaliação de arranjos mas não os aplicam (LÜBECK; WITTMAN; SILVA, 2012; JUNIOR MARINI; SILVA, 2012), a maioria propõe métodos/indicadores/técnicas e fazem aplicação destes (SHOLZ; STAUFFACHER, 2007; FROMHOLD-EISEBITH; EISEBITH, 2008; GALDAMEZ; CARPINETTI; GERÓLAMO, 2009; OLIVARES; DALCOL, 2010; TIGRE et al., 2011) e outros usam métodos/indicadores/técnicas já existentes para avaliar os arranjos (PINHEIRO; SOBREIRA; RAPINI, 2008; BRITO et al., 2010; ERLING; XIAOJIAN; ZHIGAO, 2011).

Outro ponto a ser destacado é que existe praticamente uma divisão entre os artigos no que tange ao fato do contexto e ganhos que podem advir dos arranjos produtivos contribuírem ou possam contribuir (SHOLZ; STAUFFACHER, 2007; GALDAMEZ; CARPINETTI; GERÓLAMO, 2009; BRITO et al., 2010; ERLING; XIAOJIAN; ZHIGAO, 2011; JUNIOR MARINI; SILVA, 2012) ou não terem impacto significativo (FROMHOLD-EISEBITH; EISEBITH, 2008; PINHEIRO; SOBREIRA; RAPINI, 2008; OLIVARES; DALCOL, 2010; LÜBECK; WITTMAN; SILVA, 2012) para o desenvolvimento local. O artigo de Tigre et al. (2008) não pode ser enquadrado em um dos lados da divisão, pois coloca que a contribuição para o desenvolvimento local depende da forma como os arranjos são institucionalizados e geridos, portanto depende do contexto de cada arranjo.

O Quadro 3 ilustra a análise geral descrita nos dois parágrafos anteriores. 


\begin{tabular}{|c|c|c|c|c|c|c|}
\hline Autores & 1 & 2 & 3 & 4 & 5 & 6 \\
\hline Scholz e Stauffacher (2007) & & $X$ & & $\mathrm{X}$ & & \\
\hline Fromhold-Eisebith e Eisebith (2008) & & $X$ & & & $X$ & \\
\hline Pinheiro, Sobreira e Rapini (2008) & & & $\mathrm{X}$ & & $X$ & \\
\hline Galdámez, Carpinetti e Gerolamo (2009) & & $X$ & & $\mathrm{X}$ & & \\
\hline Brito et al. (2010) & & & $X$ & $X$ & & \\
\hline Olivares e Dalcol (2010) & & $X$ & & & $X$ & \\
\hline Erling, Xiaojian e Zhigao, 2011 & & & $X$ & $\mathrm{X}$ & & \\
\hline Tigre et. al. (2011) & & $X$ & & & & $x$ \\
\hline Lübeck, Wittman e Silva (2012) & $\mathrm{X}$ & & & & $X$ & \\
\hline Junior Marini e Silva (2012) & $\mathrm{X}$ & & & $\mathrm{X}$ & & \\
\hline \multicolumn{7}{|c|}{$\begin{array}{l}\text { Legenda } \\
\text { 1- Proposta de método/indicador/técnica e não aplicação } \\
\text { 2- Proposta de método/indicador/técnica e aplicação } \\
\text { 3- Uso de método/indicador/técnica existente } \\
\text { 4- Contexto e ganhos que podem advir dos arranjos contribui para o DL } \\
\text { 5- Contexto e ganhos que podem advir dos arranjos não contribuem para o DL } \\
\text { 6- Contribuição do contexto e ganhos que podem advir dos arranjos depende do contexto }\end{array}$} \\
\hline
\end{tabular}

Quadro 3 - Análise geral dos artigos relacionados ao tema A

Fonte: Elaborado pelos autores

\section{Impacto de arranjos produtivos de setores específicos}

Procurando trazer novas evidências a respeito da natureza e importância associadas à produção de aço, Giarratani, Gruver e Jackson (2007) analisaram clusters industriais, com foco nas questões de entrada de mercado e desenvolvimento regional. Segundo os autores, os clusters podem exercer um papel importante no processo de entrada de mercado, entretanto, a contribuição para o desenvolvimento regional depende das características dos produtos e das empresas. Quando a entrada de mercado da empresa depende de outras empresas da região (ligações internas), o impacto no desenvolvimento regional é maior do que quando as empresas possuem apenas ligações externas (com empresas que estão fora da aglomeração).

Já Sable (2007) buscou avaliar o impacto dos clusters metropolitanos de biotecnologia de San Diego e Boston, no desenvolvimento econômico local, especialmente no que diz respeito ao trabalho e ao mercado imobiliário. A pesquisa mostrou que o setor de biotecnologia demanda alta qualificação, gerando pouco retorno de empregos para a economia regional. Os clusters provocaram o fenômeno de gentrificação (expulsão das populações mais carentes de áreas de grandes metrópoles e valorização imobiliária desses espaços). $\mathrm{O}$ foco na biotecnologia não é condizente com a diversidade econômica essencial para a sustentação do desenvolvimento local (leva-se muito tempo para desenvolver um produto), além disso, a biotecnologia impacta negativamente o ambiente.

Oliveira e Ali (2011) buscaram entender a dinâmica das minas de esmeralda brasileiras, localizadas em Campos Verdes/ Santa Terezinha, GO; Nova Era/Itabira, MG; e Carnaíba/Campo Formoso, BA, e seu impacto no desenvolvimento local usando o conceito de clusters. Os resultados apontaram que regiões de minas de esmeralda atraem vários imigrantes, aumentando a demanda por serviços públicos, mas o governo local não tem capacidade de provê-los devido ao pequeno retorno de impostos gerados. Existe uma lacuna entre demanda e oferta de serviços públicos, gerando vários problemas sociais. Entretanto trabalhar com conceito de clusters pode ajudar na criação de políticas para melhorar os benefícios locais, organizando os mineiros e organizações de suporte permitindo assim a realização de investimentos que possam gerar benefícios a longo prazo para as regiões.

Em relação aos artigos relacionados ao Tema B, percebe-se que o fato das empresas de aço (GIARRATANI; GRUVER; JACKSON, 2007) e as minas de esmeralda (OLIVEIRA; ALI, 2011) estarem localizadas em APLs pode 
beneficiar as empresas e o desenvolvimento local, o que depende do contexto dos produtos e das empresas, no caso do APL das empresas de aço; e da resolução de problemas sociais e criação de políticas públicas, no caso das minas de esmeralda. Já o APL de biotecnologia (SABLE, 2007), pela peculiaridade do setor, afeta negativamente o desenvolvimento local da região onde está inserido.

\section{Desenvolvimento e promoção de arranjos produtivos}

Wei, Li e Wang (2007) buscaram avançar na pesquisa de desenvolvimento regional investigando a reestruturação do distrito industrial de Wenzhou, na China. A pesquisa mostrou que o processo de reestruturação liderado pelo governo e os empresários locais que pertencem a densas redes locais, envolveu mudança institucional, modernização, diversificação e reestruturação espacial, superando os limites dos negócios familiares. Mais recentemente, o crescimento de Wenzhou tem diminuído, levando alguns a questionar o seu modelo de crescimento. Argumenta-se que o desenvolvimento de Wenzhou está em perigo devido a "trancas" regionais: relacionais, intergeracionais e estruturais. Porém a experiência de Wenzhou desafiou o conceito tradicional de distritos industriais Marshalianos, por meio do desenvolvimento regional globalizado (WEI; LI; WANG, 2007).

Mason, Castleman e Parker (2008) propuseram os conceitos de comunidades de empresas e comunidades virtuais de empresas, para descrever como pequenas e médias empresas podem atuar em rede e compartilhar conhecimento para desenvolver clusters regionais. Tais comunidades poderiam contribuir com o desenvolvimento das regiões onde os clusters estão inseridos, por meio da geração de capital intelectual; estabelecimento da cultura da inovação; criação de redes de valores; e promoção do capital social.

Através da análise do cluster da indústria intermediária de fornecedores de aço de Pittsburg; Treado e Giarratani (2008) buscaram entender a experiência e desenvolvimento desse cluster para levantar insights a serem utilizados por outros clusters industriais tradicionais como fonte de resiliência econômica e desenvolvimento regional.
Na região de Pittsburg, apesar do declínio da indústria de aço, os fornecedores sobreviveram, mantendo uma importante presença regional, servindo como fonte de exportação para fora da região. Ligações horizontais com importantes parceiros regionais (cooperação para buscar oportunidades de mercado, desenvolvimento de produtos, resolução de problemas) foram fundamentais para a sustentação do cluster. Os fornecedores locais também apresentaram grande capacidade na transferência de conhecimento tecnológico para a diferenciação de produto, ampliando sua base de clientes e aumentando a rentabilidade. Os laços no cluster podem ser estreitados por meio de políticas públicas, associações profissionais e universidades (TREADO; GIARRATANI, 2008).

Czepczynski (2009) foca em um aspecto mais específico dos arranjos produtivos e seu impacto no desenvolvimento regional: a cooperação. O autor buscou apresentar alguns obstáculos chave e condições gerais que moldam a cooperação regional entre municipalidades da terceira maior aglomeração da Polônia em Gdansk, também conhecida com Tri-City.

Verificou-se que a concorrência econômica e as práticas liberais de governança pós-1990 melhoraram o isolacionismo e rivalidade entre as localidades da aglomeração. Porém, as demandas públicas para cooperação são principalmente limitadas aos aspectos práticos do transporte público. $\mathrm{O}$ principal obstáculo para a cooperação não é a cooperação em si, mas sim como colocá-la em prática sem perda de poder das localidades. As ambições e aspirações de atores políticos locais e as profundas antipatias e aversões entre as principais cidades da aglomeração, são o fator principal por trás desse obstáculo (CZEPCZYNSKI, 2009).

Franz e Horrych (2010) discutiram a questão do desenvolvimento de aglomerações urbanas, através da elaboração de um quadro teórico abordando a cooperação entre municípios na perspectiva da economia regional e da ciência política. A teoria é aplicada à região metropolitana denominada "Triângulo Saxônico", na Alemanha. Os resultados mostraram que vários fatores (falta de confiança, situações ganha-perde, relações não estáveis) prejudicam a cooperação entre os atores da 
região, e que políticas públicas deveriam ser tomadas para reverter essa situação, favorecendo a economia e o desenvolvimento local.

Parker (2010) procurou compreender o processo de formação e evolução de clusters, por meio da mudança transformativa e adaptativa, obtida através da conscientização e reflexão dos atores públicos e privados. Analisando clusters tecnológicos de diferentes tamanhos, idade e localidade (Sofia Antipolis, na França, e Hsinchu Science Park, em Taiwan) os autores concluíram que a mudança institucional que envolve a transformação de uma cidade/região não depende de uma crise induzida de forma exógena, e sim pode envolver o acesso a novas ideias e visões de empresários e atores políticos públicos e privados.

Segundo Parker (2010), a mudança transformadora pode envolver um processo pelo qual as visões dos empresários sejam viáveis e estes obtenham recursos de instituições de apoio para suportar a transformação regional. Além disso, os clusters podem evoluir e mudar através de um processo reflexivo de aprendizagem estratégica em que os atores identificam problemas na região, reconfigurando-a para novos fins, atendendo assim suas necessidades e contribuindo para o desenvolvimento regional.

Já Chou et al. (2011), consideraram o quadro analítico de acoplamento estratégico para analisar o caso do cluster chinês de semicondutores de Wuxi. Eles investigaram a formação, dinâmica e efeitos gerados por firmas domésticas, ao invés de estrangeiras, no cluster. As evidências mostraram que, apesar de a China ter uma estratégia de equilíbrio entre promoção de investimento estrangeiro e aceleração do desenvolvimento das firmas locais, o surgimento e crescimento do cluster de Wuxi não se deve ao investimento estrangeiro. Em vez disso, resulta da interação dinâmica de vários elementos como: a mediação do Estado, a comunidade técnica transnacional chinesa e as empresas nacionais.

Chou et al (2011) ainda colocam que efeitos de spillover tecnológico derivados de instituições de pesquisa governamentais, assim como cooperação e competição mútuas na rivalidade tecnológica entre empresas domésticas, são considerados como elementos importantes para o desenvolvimento do cluster e do desenvolvimento regional.
A pesquisa de Ranjith e Widner (2011) buscou avaliar a deslocalização da indústria de vestuário de Colombo, no Sri Lanka, da região metropolitana para áreas suburbanas, visando alcançar economias de aglomeração, como estratégia para o desenvolvimento regional.

Os autores concluíram que muitas condições necessárias para o desenvolvimento regional através da deslocalização da indústria do vestuário ainda não estão presentes, sendo preciso: investir em novas tecnologias; atualizar as competências dos trabalhadores; melhorar processos, a organização da produção e a qualidade dos produtos; proteger os direitos humanos dos cidadãos, manter a estabilidade política, melhorar as condições de trabalho. Isso contribuiria para o aumento da produtividade e competitividade e a melhoria da qualidade de vida no trabalho, nas empresas do vestuário no Sri Lanka (RANJITH; WIDNER, 2011).

Bellandi e Lombardi (2012), por sua vez, analisaram quatro mercados especializados (têxtil, couro, embutidos e hardware) pertencentes à Província de Zejiang para compreender seu modelo de desenvolvimento (baseado na interação entre mercados especializados e clusters industriais), e entender qual é o papel que desempenham tais mercados, considerando o referido modelo e as lições que podem ser aprendidas por meio de uma perspectiva político-econômica comparativa.

Os resultados apontaram que, junto com as tradições locais e habilidades, na produção de artesanato e comércio, mercados especializados acompanharam a decolagem de clusters industriais após o chamado "modelo Zhejiang" de desenvolvimento. A partir de uma perspectiva de economia política comparativa, a interação das variáveis econômicas e sociopolíticas em nível local ocorrida na província de Zhejiang destaca o papel da ação coletiva (entre instituições locais e empreendedores) no processo de desenvolvimento local sustentado, incorporado pela tradição. O papel dos mercados especializados ainda é crucial hoje em dia como um canal eficaz para a distribuição de produtos e como um meio de coordenação de produções fragmentadas na China e no exterior (BELLANDI; LOMBARDI, 2012).

Os autores Cunha, Passador e Passador (2012) verificaram se a presença de agentes 
públicos e privados é essencial para a formação de redes no APL de calçados de Birigui, destacando que o associativismo promovido por esses agentes é fundamental para impulsionar o desenvolvimento local. A pesquisa apontou que existe um legado regional que sustenta o know-how da indústria de calçados, mas o interesse dos participantes do APL é principalmente comercial, não havendo um capital social desenvolvido, que crie associações provenientes de vontades próprias das organizações. A presença de um agente intermediador por si só não garante a formação de laços de proximidade entre os membros da rede, mas é importante para unir interesses de curto prazo das empresas (ganhos econômicos e comerciais).

Quandt (2012) procurou investigar de que forma as interações dos agentes que atuam em APLs contribuem para o desenvolvimento de inovações em produtos e processos, e para o aumento da competitividade e desenvolvimento econômico local. $\mathrm{O}$ autor realizou um estudo no APL de malharias de Imbituva, PR, que revelou serem pouco expressivas as inovações no arranjo, tendo impacto limitado nas organizações. A pesquisa ainda mostrou uma relação positiva entre intensidade de interações, propensão a inovar e desempenho dos negócios, porém tais aspectos ainda são insipientes. As empresas mais antigas apresentaram um padrão mais intenso de interações. Destacou-se a importância de iniciativas para estimular a inovação e o aprendizado por interação entre empresas e instituições do APL, visando ao seu desenvolvimento (QUANDT, 2012).

A análise geral dos artigos relacionados ao tema $C$ permite que algumas observações sejam feitas. Dois artigos tratam de forma mais geral (WEI; LI; WANG, 2007; TREADO; GIARRATANI, 2008) a questão do desenvolvimento e evolução dos APLs e seu impacto no desenvolvimento local, e os demais (nove artigos) tratam aspectos mais específicos como: redes (MASON; CASTLEMAN; PARKER, 2008; CUNHA;
PASSADOR; PASSADOR, 2012); cooperação (CZEPCZYNSKI, 2009; FRANZ; HORRYCH, 2010); mudança (PARKER, 2010); deslocalização de empresas (RANJITH; WIDNER, 2011); inovação (QUANDT, 2012); interações / realização de ações coletivas entre agentes e empresas do arranjo (CHOU et al., 2011; BELLANDI; LOMBARDI, 2012; CUNHA; PASSADOR; PASSADOR, 2012; QUANDT, 2012).

Pode-se também relacionar se os resultados dos estudos que apontaram ou não para uma contribuição do contexto estudado nos arranjos para o desenvolvimento local, sendo que houve praticamente uma divisão dos estudos (Quadro 3). Seis artigos (WEI; LI; WANG, 2007; MASON; CASTLEMAN; PARKER, 2008; TREADO; GIARRATANI, 2008; PARKER, 2010; CHOU et al., 2011; BELLANDI; LOMBARDI, 2012) revelaram que houve contribuição para o desenvolvimento local, já os demais artigos (CZEPCZYNSK, 2009; FRANZ; HORRYCH, 2010; RANJITH; WIDNER, 2011; CUNHA; PASSADOR; PASSADOR, 2012; QUANDT, 2012) indicaram pouca ou ausência de contribuição.

O incentivo a iniciativas, através de políticas públicas e/ou ações privadas foi um dos pontos de destaque para a melhoria da contribuição dos APLs, respeitando o foco de estudo de cada artigo, para o desenvolvimento local (TREADO; GIARRATANI, 2008; FRANZ; HORRYCH, 2010; RANJITH; WIDNER, 2011; QUANDT, 2012).

O Quadro 3 relaciona os autores de acordo com a abordagem mais geral ou mais específica (dividida nos aspectos enfatizados nos artigos) e também se os resultados apontaram uma contribuição ou não para o desenvolvimento local. A questão da interação e realização de ações coletivas entre agentes e empresas dos arranjos foi a abordagem específica de maior representatividade, seguida pela cooperação. Artigos como o de Cunha, Passador e Passador (2012) e Quandt (2012) focaram duas abordagens específicas, cada um. 


\begin{tabular}{|c|c|c|c|c|c|c|c|c|c|}
\hline \multirow[t]{2}{*}{ Autores } & \multirow[t]{2}{*}{ AG } & \multicolumn{6}{|c|}{ AE } & \multirow{2}{*}{$\begin{array}{l}\text { Contribui } \\
\text { para o DL }\end{array}$} & \multirow{2}{*}{$\begin{array}{c}\text { Contribui pouco/ } \\
\text { não contribui } \\
\text { para o DL }\end{array}$} \\
\hline & & 1 & 2 & 3 & 4 & 5 & 6 & & \\
\hline Wei, Li e Wang (2007) & $\mathrm{X}$ & & & & & & & $X$ & \\
\hline Mason, Castleman e Parker (2008) & & $\mathrm{X}$ & & & & & & $X$ & \\
\hline Treado e Giarratani (2008) & $X$ & & & & & & & $X$ & \\
\hline Czepczynski (2009) & & & $\mathrm{X}$ & & & & & & $\mathrm{X}$ \\
\hline Franz e Hornych (2010) & & & $\mathrm{X}$ & & & & & & $\mathrm{X}$ \\
\hline Parker (2010) & & & & $X$ & & & & $X$ & \\
\hline Chou et al. (2011) & & & & & & & $\mathrm{X}$ & $X$ & \\
\hline Ranjith e Widner (2011) & & & & & $X$ & & & & $\mathrm{X}$ \\
\hline Bellandi e Lombardi (2012) & & & & & & & $X$ & $X$ & \\
\hline Cunha, Passador e Passador (2012) & & $\mathrm{X}$ & & & & & $\mathrm{X}$ & & $X$ \\
\hline Quandt (2012) & & & & & & $\mathrm{X}$ & $\mathrm{X}$ & & $X$ \\
\hline
\end{tabular}

Quadro 4 - Abordagens (geral ou específica) relacionadas aos artigos do tema C.

Fonte: Elaborado pelos autores

\section{Considerações Finais}

O presente artigo utilizou o método de revisão sistemática para avaliar a produção bibliográfica a respeito do desenvolvimento local em arranjos produtivos locais, permitindo assim uma pesquisa com maior rigor metodológico, conforme Tranfield, Denyer e Smart (2003) e, portanto, atingindo ao objetivo proposto.

A avaliação permitiu o agrupamento dos 24 artigos constituintes do corpus da pesquisa nos seguintes temas: A) Uso de indicadores, métodos e técnicas para avaliação de arranjos produtivos; B) Impacto de arranjos produtivos de setores específicos; C) Desenvolvimento e promoção de arranjos produtivos, sendo feito um detalhamento e análise geral dos artigos dentro de cada um dos temas.

É importante destacar que tanto no tema $\mathrm{A}$, quanto no tema $\mathrm{C}$, no que tange à contribuição dos arranjos para o desenvolvimento local, cerca de metade dos artigos apontou contribuição, e a outra metade apontou pouca ou nenhuma contribuição. Isso mostra que a capacidade que os arranjos produtivos têm de fomentar o desenvolvimento local, segundo a literatura (ISBASOIU, 2007; OLIVARES; DALCOL, 2010) não pode ser desconsiderada, mas que há ainda medidas a serem tomadas, por exemplo, no que tange a ações e iniciativas viabilizadas por instituições públicas e priva- das em parceria com atores e empresas dos arranjos, para que tal contribuição seja efetiva.

Como pesquisa futura, sugere-se a realização de revisão sistemática para os temas desenvolvimento local e arranjos produtivos locais, separadamente, permitindo assim conhecer e analisar os vários assuntos que vêm sendo pesquisados relacionados a esses temas.

\section{Agradecimentos}

Os autores agradecem ao $\mathrm{CNPq}$ (Conselho Nacional de Desenvolvimento Científico e Tecnológico) por ter financiado e possibilitado esta pesquisa.

\section{Referências}

BELLANDI, M.; LOMBARDI, S. Specialized markets and chinese industrial clusters: the experience of Zhjiang Province. China Economic Review, Ohio, v. 23, p. 626-638, 2012.

BRITO, E. P. Z.; BRITO, L. A. L.; PORTO, E. C.; SZILAGYI, M. E. A relação entre aglomeração produtiva e crescimento: aplicação de um modelo multinível ao setor industrial paulista. Revista de Administração Contemporânea, Curitiba, v. 14, n. 4, p. 615-632, jul./ ago. 2010.

CASSIOLATO, J. E.; LASTRES, H. M. M. O foco em arranjos produtivos e inovativos locais de micro e pequenas empresas. In: LASTRES, H. M. M.; CASSIOLATO, J. E.; MACIEL, M. L. (Org.). Pequena empresa: cooperação e desenvolvimento local. Rio de Janeiro: Relume Dumará, 2003, p. 21-34. 
CHOU, T. L.; CHING, C. H.; FAN, S. M.; CHANG, J. Y. Global linkages, the chinese high-tech community and industrial cluster development: the semiconductor industry in Wuxi, Jingsu. Urban Studies, Glasgow, v. 48, n. 14, p. 3019-3042, nov. 2011.

CZEPCZYNSKI, M. Regionalisation in the Gdansk area: virtual disintegration and functional cooperation. International Journal of Public Sector Management, Bingley, $\mathrm{v}$. 22, n. 3, p. 249-259, 2009.

CUNHA, J. L. C.; PASSADOR, J. L.; PASSADOR, C. S. A presença de agentes intermediadores na formação de redes interorganizacionais: uma análise sob a perspectiva temporal. Cadernos Ebape, Rio de Janeiro, v. 10, n. 1, p. 108-128, mar. 2012.

ERLING, Li.; XIAOJIAN, Li; ZHIGAO, Liu. Relationships and evolving networks of rural manufacturing clusters: a case study in Yucheng County, Henan Province of China. Chinese Geographical Science, Beijing, v. 21, n. 3, p. 364-376, 2011.

FRANZ, P.; HORNYCH, C. Political institutionalisation and economic specialization in polycentric metropolitan regions: the case of east German 'saxony triangle'. Urban Studies, Glasgow, v. 47, n. 12, p. 2665-2682, nov. 2010.

FROMHOLD-EISEBITH, M.; EISEBITH, G. Looking behind facades: evaluating effects of (automotive) cluster promotion. Regional Studies, Seaford, v. 42, n. 10, p. 1343-1356, dec. 2008.

FUCHS, S. C.; PAIM, B. S. Revisão sistemática de estudos observacionais com metanálise. Revisa HCPA, Porto Alegre, v. 30, n. 3, p. 294-301, 2010.

GALDÁMEZ, E. V. C.; CARPINETTI, L. C. R.; GEROLAMO, M. C. Proposta de um sistema de avaliação do desempenho para arranjos produtivos locais. Gestão $\mathcal{E}$ Produção, São Carlos, v. 16, n. 1, p. 133-151, jan./mar. 2009.

GIARRATANI, F. G.; GRUVER, G.; JACKSON, R. Clusters, agglomeration, and economic development potential: empirical evidence based on the advent of slab casting by U.S. steel minimils. Economic Development Quarterly, Kalamazoo, v. 21, n. 2, p. 148-164, may. 2007.

ISBASOIU, G. M. Industrial clusters and regional development. The case of Timesoara and Montebelluna. Munich Personal Research Archive, Munich, n. 5037, nov. 2007.

JUNIOR MARINI, M.; SILVA, C. L. Desenvolvimento regional e arranjos produtivos locais: uma abordagem sob a ótica interdisciplinar. Revista Brasileira de Gestão e Desenvolvimento Regional, Taubaté, v. 8, n. 2, p. 107-129, maio/ago. 2012

LÜBECK, R. M.; WITTMANN, M. L.; SILVA, M. S. Afinal, quais variáveis caracterizam a existência de cluster, arranjos produtivos locais (APLs) e dos sistemas locais de produção e inovação (SLPIs)? Revista Ibero-Amerciana de Estratégia, São Paulo, v. 11, n. 1, p. 120-151, jan./abr. 2012.

MASON, C.; CASTLEMAN, T.; PARKER, C. Communities of enterprise: developing regional SMEs in the knowledge economy. Journal of Enterprise Information Management, Bingley, v. 21, n. 6, p. 571-584, 2008.

MATTOS, S. M. C. S. Arranjos produtivos locais como estratégia para o desenvolvimento local: o caso de Maracás. Sitientibus, Feira de Santana, n. 39, p. 131-167, jul./dez. 2008.
OLIVARES, G. P.; DALCOL, P. R. T. Proposta de um sistema de indicadores para medir o grau de contribuição dos aglomerados produtivos para o desenvolvimento local e regional. Revista Brasileira de Gestão e Desenvolvimento Regional, Taubaté, v. 6, n. 2, p. 188-218, maio/ago. 2010.

OLIVEIRA, J. A. P; ALI, S. H. Gemstone mining as a development cluster: a study of Brazil's emerald mines. Resources Policy, Philadelphia, v. 36, p. 132-141, 2011.

PARKER, R. Evolution and change in industrial clusters: an analysis of Hsinchu and Sophia Antipolis. European Urban and Regional Studies, Thousand Oaks, v. 17, n. 3, p. 245-260, 2010.

PINHEIRO, A. M.; SOBREIRA, L. M. G.; RAPINI, M. S. Aglomerações produtivas no estado do Pará: uma proposta de análise para a construção civil. Revista Brasileira de Gestão e Desenvolvimento Regional, Taubaté, v. 4, n. 1, p. 24-56, jan./abr. 2008.

QUANDT, C. O. Redes de cooperação e inovação localizada: estudo de caso de um arranjo produtivo local. Revista de Administração e Inovação, São Paulo, v. 9, n. 1, p. 141-166, jan./mar. 2012.

RANJITH, J. G. S.; WIDNER, B. Sri Lanka's garment industry: prospects for agglomeration, challenges and implications for regional development. South Asian Journal of Management, Hyderabad, v. 18, n. 4, p. 7-22, oct./dec. 2011.

SABLE, M. The impact of the biotechnology industry on local economic development in the Boston and San Diego metropolitan areas. Technological Forecasting $\mathcal{E}$ Social Change, Philadelphia, v. 74, p. 36-60, 2007.

SCHOLZ, R. W.; STAUFFACHER, M. Managing transition in clusters: area development negotiations as a tool for sustaining traditional industries in a Swiss prealpine region. Environment and Planning A, London, v. 39, p. 2518-2539, 2007.

TRANFIELD, D.; DENYER, D.; SMART, P. Towards a methodology for developing evidence-informed management knowledge by means of systematic review. British Journal of Management, London, v. 14, p. 207-222, 2003.

TREADO, C. D.; GIARRATANI, F. Intermediate steelindustry suppliers in the Pittsburgh region: a clusterbased analysis of regional economic resilience. Economic Development Quarterly, Kalamazoo, v. 22, n. 1, p. 63-75, feb. 2008

TIGRE, P. B.; LA ROVERE, L. R.; TEIXEIRA, F. L.; LÓPES, A.; RAMOS, D.; BERCOVICH, N. Knowledge cities: a taxonomy for analyzing software and information service clusters. Revista de Administração de Empresas, São Paulo, v. 51, n. 1, p. 15-26, jan/fev. 2011.

ULTRAMARI, C.; DUARTE, F. Desenvolvimento local e regional. 2. ed. Curitiba: Ibpex, 2011.

WEI, Y. D.; LI, W.; WANG, C. Restructuring industrial districts, scaling up regional development; a study of the Wenzhou Model, China. Economic Geography, Worcester, v. 83, n. 4 , p. 421-444, oct. 2007. 